\title{
STAT3 can serve as a hit in the process of malignant transformation of primary cells
}

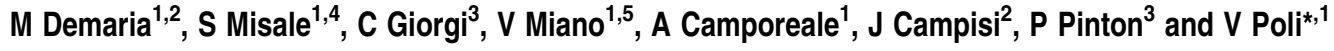

The transcription factor signal transducer and activator of transcription 3 (STAT3) acts downstream of many pro-oncogenic signals, including cytokines, growth factors and oncogenes, and is accordingly constitutively active in a wide variety of tumors that often become addicted to it. Moreover, STAT3 is a key player in mediating inflammation-driven tumorigenesis, where its aberrant continuous activation is typically triggered by local or systemic production of the pro-inflammatory cytokine IL-6. We recently showed that mouse embryonic fibroblasts (MEFs) derived from STAT3C k/in mice, which express physiological levels of the constitutively active mutant STAT3C, display features of transformed cells such as increased proliferation, resistance to apoptosis and senescence, and aerobic glycolysis. Here, we show that pre-existing constitutively active STAT3 is sufficient to prime primary MEFs for malignant transformation upon spontaneous immortalization. Transformation is strictly STAT3dependent and correlates with high resistance to apoptosis and enhanced expression of anti-apoptotic/pro-survival genes. Additionally, hypoxia inducible factor (HIF)- $1 \alpha$ level is elevated by twofold and contributes to STAT3 oncogenic activity by supporting high rates of aerobic glycolysis. Thus, constitutively active STAT3, an accepted essential factor for tumor growth/ progression, can also act as a first hit in multistep carcinogenesis; this ability to predispose cells to malignant transformation may be particularly relevant in the pro-oncogenic niche represented by chronically inflamed tissues.

Cell Death and Differentiation (2012) 19, 1390-1397; doi:10.1038/cdd.2012.20; published online 9 March 2012

Signal transducers and activators of transcription (STAT) factors mediate the signaling downstream of cytokine and growth factor receptors. ${ }^{1,2}$ Once activated by tyrosinephosphorylation by receptor-associated JAK kinases, STATs concentrate into the nucleus and regulate the expression of target genes. ${ }^{3}$ STAT3 can be activated by a wide variety of cytokines and growth factors and by a number of oncogenes, ${ }^{1}$ and is accordingly constitutively phosphorylated on tyrosine in many tumors that often become addicted to its activity. ${ }^{1,2,4}$ Indeed, STAT3 is required for cell transformation downstream of $\mathrm{v}$-Src and many other oncogenes, ${ }^{4-6}$ and overexpression of a constitutively active mutant form can transform immortalized fibroblasts and epithelial cells. ${ }^{7,8}$ Even though STAT3 is often referred to as an oncogene, only recently a naturally activating mutation has been found in human hepatocellular adenomas, ${ }^{9}$ and its aberrant activity in tumors mostly occurs downstream of multiple activated pro-oncogenic pathways. Importantly, STAT3 is a key player in mediating inflammationdriven tumorigenesis, being constitutively activated by chronically high levels of the pro-inflammatory cytokine IL-6. ${ }^{10}$ In tumors, STAT3 is known to enhance cell survival and proliferation and to promote immune escape and angiogenesis, invasion and metastasis. ${ }^{11,12}$
In an effort to characterize pro-oncogenic functions of continuous, weak STAT3 activation, we have recently generated knock-in mice expressing physiological levels of the constitutively active STAT3C mutant form. ${ }^{13}$ We have shown that constitutively active STAT3 acts as a master regulator of cell metabolism, promoting aerobic glycolysis and downregulating mitochondrial activity by its canonical/nuclear functions both in $\mathrm{Stat3}^{\mathrm{C} / \mathrm{C}}$ mouse embryonic fibroblasts (MEFs) and in STAT3-addicted tumoral cells. ${ }^{14}$ On the other hand, mitochondrially localized STAT3 was recently demonstrated to have a role in preserving mitochondrial respiratory activity by its serine-phosphorylated form. ${ }^{15,16}$ Both activities contribute to malignant transformation downstream of distinct signals, which promote STAT3 phosphorylation on either tyrosine or serine thus activating nuclear or mitochondrial STAT3 (nSTAT3 and mSTAT3). ${ }^{17}$ Although aerobic glycolysis induced by nuclear STAT3 is dependent on the transcriptional induction of the oxygen-sensor hypoxia inducible factor (HIF)- $1 \alpha$, downregulation of mitochondrial function is HIF- $1 \alpha$-independent. ${ }^{14}$ HIF-1 is composed of a constitutively expressed $\beta$ subunit and an $\mathrm{O}_{2}$-regulated $\alpha$ subunit, ${ }^{18}$ which undergoes $\mathrm{O}_{2}$-dependent hydroxylation leading to its proteasomal degradation. ${ }^{19}$ Under hypoxic

${ }^{1}$ Molecular Biotechnology Center and Department of Genetics, Biology and Biochemistry, University of Turin, Turin, Italy; ${ }^{2}$ Buck Institute for Research on Aging, Novato, CA, USA and ${ }^{3}$ Department of Experimental and Diagnostic Medicine, Section of General Pathology, Interdisciplinary Center for the Study of Inflammation (ICSI), Laboratory for Technologies of Advanced Therapies (LTTA), University of Ferrara, Ferrara, Italy

*Corresponding author: V Poli, Molecular Biotechnology Center and Department of Genetics, Biology and Biochemistry, University of Turin, Via Nizza 52, Torino 10126, Italy. Tel: + 3911 6706428; Fax: + 3911 6706432; E-mail: valeria.poli@unito.it

${ }^{4}$ Present address: Laboratory of Molecular Genetics, Institute for Cancer Research and Treatment, Candiolo, Italy.

${ }^{5}$ Present address: Center for Molecular Systems Biology and Department of Oncological Sciences, University of Turin, Turin, Italy.

Keywords: STAT3; HIF-1 $\alpha$; aerobic glycolysis; tumorigenesis; apoptosis; 3T3 MEFs

Abbreviations: STAT3, signal transducer and activator of transcription 3; HIF, hypoxia inducible factor; bcl-2, B-cell lymphoma 2; bcl-2l1, B-cell lymphoma 2 like-1; bcl-3, B-cell lymphoma 3; birc5, baculoviral inhibitor of apoptosis repeat-containing 5; Sco2, synthesis of cytochrome c oxidase 2; Tigar, Tp53-induced glycolysis and apoptosis regulator; MEF, mouse embryonic fibroblast; qRT-PCR, quantitative reverse-transription polymerase chain reaction

Received 23.8.11; revised 02.2.12; accepted 02.2.12; Edited by RA Knight; published online 09.3.12 
conditions, hydroxylation is inhibited and $\mathrm{HIF}-1 \alpha$ rapidly accumulates and dimerizes with $\mathrm{HIF}-1 \beta$, becoming transcriptionally active. Oncogenic pathways can lead to HIF- $1 \alpha$ accumulation even under normoxia, ${ }^{18}$ and its enhanced activity correlates with poor prognosis in human cancer such as those of the breast, prostate and melanomas. ${ }^{20-22} \mathrm{HIF}-1 \alpha$ target genes control both oxygen delivery and usage by induction of angiogenesis and glycolysis, respectively, and this factor is one of the key players in inducing the Warburg's effect, that is, the ability of most cancer cells to undergo aerobic glycolysis. ${ }^{23,24}$

Mouse embryonic cells (MEFs) can be spontaneously immortalized via regular passages during which, after undergoing a proliferative senescence crisis, a few immortalized cells emerge (the 3T3 protocol). ${ }^{25}$ 3T3-immortalized MEFs are not tumorigenic but, in contrast to their primary counterparts, can undergo full transformation when challenged with a single oncogene. ${ }^{26}$ Primary MEFs derived from STAT3C mice (Stat3 ${ }^{C / C}$ MEFs) are not spontaneously transformed but show pre-oncogenic properties, such as increased proliferation, resistance to apoptosis and senescence and increased aerobic glycolysis with enhanced glucose dependence. ${ }^{14}$ Here we show that spontaneous immortalization via the 3T3 protocol leads to complete transformation, demonstrating that continuous STAT3 activity can act as a first hit in tumorigenesis. This has important implications for understanding the mechanisms leading to inflammation-driven cancer, where chronically active STAT3 precedes tumor transformation.

\section{Results}

Immortalization triggers tumorigenic transformation in Stat3 ${ }^{C / C}$ MEFs. MEFs derived from two individual Stat $3^{C / C}$ or Stat3 ${ }^{W T / W T}$ embryos were subjected to the 3Т3 spontaneous immortalization protocol. $^{25}$ Compared with wild-type cells, Stat ${ }^{C / C}$ MEFs underwent a delayed and much shorter proliferative crisis, which peaked at passage (P) 16 as compared with P10 of their wild-type counterparts (Figure 1a). Cells never completely ceased duplicating and a rapidly proliferating population arose earlier than in Stat3 ${ }^{W T W T}$ MEFs (P20 versus P24). Immortalized cell lines were completely established after 26 passages for both genotypes (two independent experiments). Similar to what we observed in the primary cells, 3T3-immortalized Stat3 ${ }^{C / C}$ MEFs displayed increased expression of the STAT3 canonical target gene Socs3, confirming higher STAT3 transcriptional activity, and proliferated much faster than their wild-type counterparts (Figures $1 \mathrm{~b}$ and $\mathrm{c}$ ). However, in contrast to primary Stat3 ${ }^{C / C}$ MEFs, both of the immortal Stat ${ }^{C / C}$ cell lines $(\mathrm{C} 1$ and $\mathrm{C} 2)$ acquired the ability to grow in multi-layers, a feature typical of transformed cells (Figure 2a). Further, both $\mathrm{C} 1$ and $\mathrm{C} 2$ were able to give rise to foci in a classical focus-forming assay (FFA), whereas immortal Stat3 ${ }^{W T / W T}$ cells were never able to do so (Figure 2b). Immortal Stat ${ }^{C / C}$ cells were also capable of anchorage-independent growth, giving rise to colonies in soft agar (Figure 2c). The ultimate test for in vitro tumor transformation is considered the ability to grow in vivo in immune-compromised mice. Immortal Stat3 ${ }^{C / C}$ or Stat3 ${ }^{W T W T}$

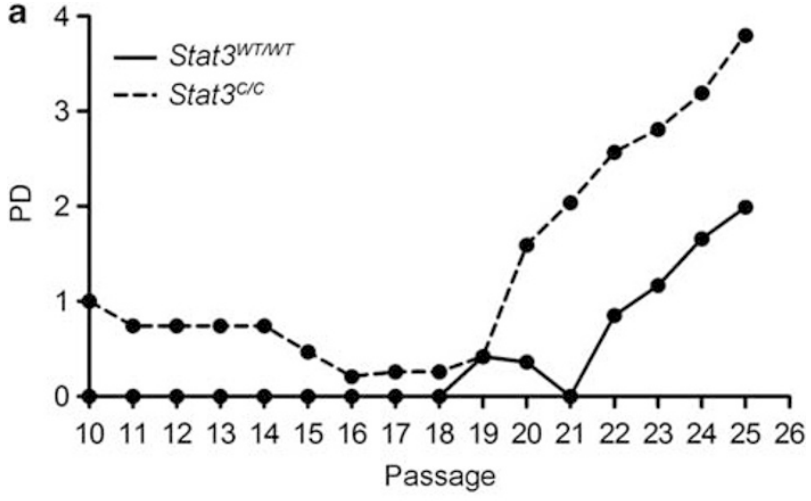

b

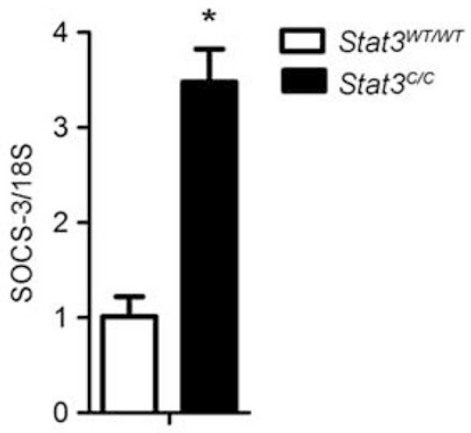

C

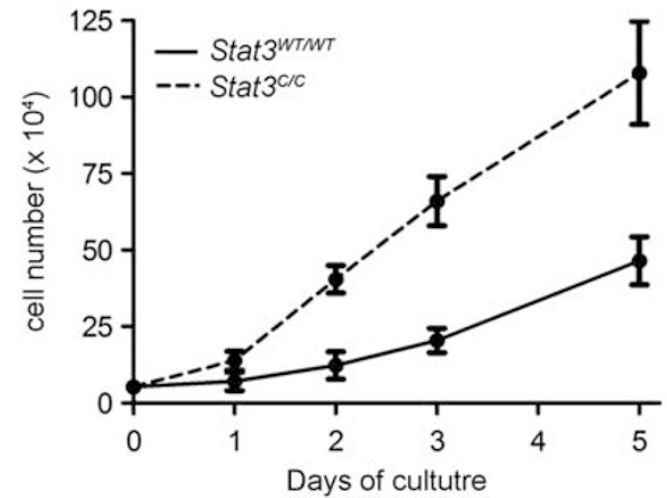

Figure 1 Immortalization and characterization of the Stat3 ${ }^{C / C}$ MEFs. (a) MEFs were derived from E14 embryos of the indicated genotypes and re-seeded at a density of $2 \times 10^{5}$ cells per $10 \mathrm{~cm}^{2}$ every 3 days as described in the Materials and Methods section. Population doubling (PD) was calculated at each passage and is shown from P10 to P25. (b) Socs3 mRNA levels were measured by Taqman RT-PCR in immortalized Stat3 ${ }^{C / C}$ (black bars) or ${ }^{W T W T}$ (white bars) MEFs. $N=4$. ${ }^{*} P<0.01$. (c) Immortalized $S$ tat ${ }^{C / C}$ MEFs display increased proliferation rates. In all, $10^{4}$ cells were plated and cells were counted every day for 5 days

MEFs $\left(10^{6}\right)$ were inoculated subcutaneously (s.c.) into nude mice. After $10-12$ days the Stat ${ }^{C / C}$ cells gave rise to palpable tumors, which reached $1 \mathrm{~cm}$ diameter by 6 weeks, at which time the mice were killed (Figure 2e). As expected, Stat3 ${ }^{W T / W T}$ cells were never able to give rise to tumors.

Thus, immortal Stat3 ${ }^{C / C}$ cells display the hallmark of fully malignant tumor cells, suggesting that constitutively active STAT3 can indeed act as a first hit in tumorigenesis. Interestingly, the transformed phenotype remained completely dependent on STAT3 activity, as shown by the observation that Stat3 silencing completely abolished in vivo growth (Figures 2d and e). 
a

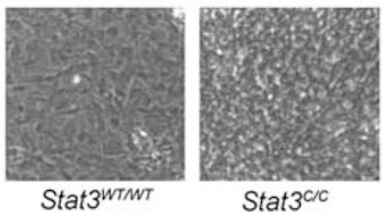

d

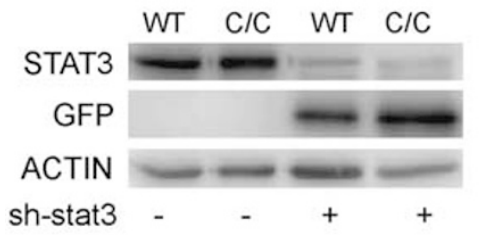

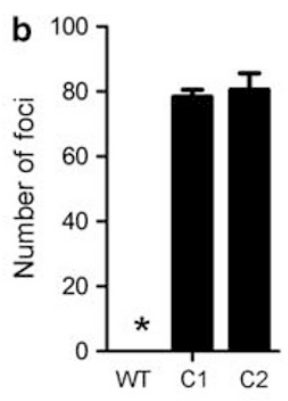
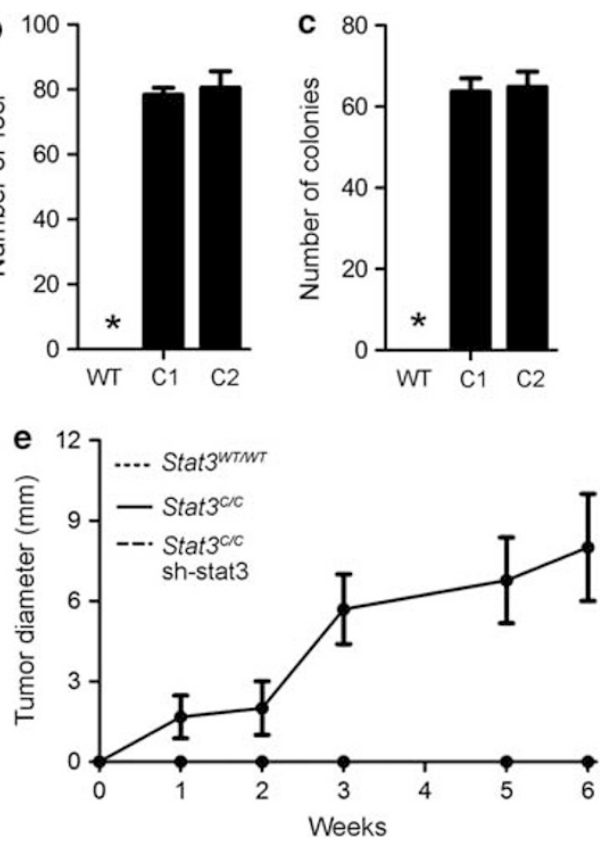

Figure 2 Transformed features of immortalized Stat3 ${ }^{C / C} \mathrm{MEFs}$. (a) Phase contrast pictures showing multi-layer growth of Stat3 ${ }^{\mathrm{C} / \mathrm{C}} \mathrm{MEFs}$. Magnification $\times 10$. (b) FFA. In all, $30 \times 10^{4}$ cells were seeded and the number of colonies scored after 15 days using methyl violet. WT, Stat3 ${ }^{\text {WTWT}} ; \mathrm{C} 1=$ Stat3 ${ }^{\mathrm{C} / C}$ line no. I, C2 $=$ Stat3 ${ }^{\mathrm{C} / \mathrm{C}}$ line no. II. $N=3$. ${ }^{*}$ Not detectable. (c) Soft-agar growth. In all, $2 \times 10^{4}$ cells (as in b) were seeded in soft agar and colony number evaluated after 30 days. $N=3$. (d) STAT3 silencing. STAT3 levels were measured by western blot in cells of the indicated genotypes, plus or minus lentiviral-mediated STAT3 silencing (sh-stat3). ACTIN was used as an internal control, and GFP to assess lentiviral infection. (e) In vivo tumor growth. In all, $10^{6}$ cells (Stat3 ${ }^{C / C}$, Stat3 ${ }^{W T W T}$ or Stat3 ${ }^{\text {C/C }}$ silenced for STAT3 - sh-stat3) were inoculated s.c. in the flank in nude mice $(N=6)$, and tumor growth was monitored each week for 6 weeks

Resistance to apoptosis of the immortalized Stat3 ${ }^{c / C}$ MEFs. STAT3 has been linked to apoptosis resistance in many tumors and experimental systems, including cells overexpressing STAT3C. ${ }^{7}$ In order to assess the ability of the transformed $S$ tat $^{C / C}$ cells to survive apoptotic stimuli, immortal $S t a t 3^{C / C}$ and Stat3 ${ }^{W T / W T}$ cells were exposed to UV-B light, followed by apoptotic index assessment using a cytofluorimeter and Annexin V staining. Indeed, and similar to the primary cells of the same genotype, immortal Stat $3^{C / C}$ cells were significantly protected from apoptotic cell death compared with their wild-type counterparts (Figure 3a). In order to evaluate activation of the pro-apoptotic caspase cascade, the levels of active caspase- 3 were measured by either immunofluorescence or western blotting after UV irradiation (Figure $3 \mathrm{~b}$ and Supplementary Figure 1a). Caspase-3 activation was lower in Stat3 ${ }^{\mathrm{C} / C}$ cells, confirming a general downregulation of the apoptotic cascade. Immortal Stat ${ }^{C / C}$ cells were also protected from starvation-induced apoptosis (Supplementary Figure 1b), supporting the idea that STAT3 acts as an anti-apoptotic factor in response to different pro-apoptotic stimuli.

As STAT3 was proposed to directly regulate a number of anti-apoptotic genes, we assessed a panel of pro- and antiapoptotic mRNAs by quantitative reverse-transription polymerase chain reaction (qRT-PCR) (data not shown). Immortal Stat ${ }^{C / C}$ cells expressed higher levels of the anti-apoptotic mRNAs bcl-2 (B-cell lymphoma 2), bcl2-l1 (B-cell lymphoma 2 like-1) and bcl-3 (B-cell lymphoma 3), all known STAT3 targets, which could well mediate the observed resistance to apoptosis (Figure 3c). Moreover, the levels of another direct STAT3 transcriptional target, the inhibitor of apoptosis gene family member baculoviral inhibitor of apoptosis repeatcontaining 5 (birc-5)/survivin, were significantly higher upon UV irradiation in the $S t a t 3^{C / C}$ than in the wild-type cells (Figure 3d). Birc-5/survivin is known to be normally expressed at low levels but to be strongly induced by apoptotic stimuli, thus protecting cells from programmed cell death. Constitutively active STAT3 is apparently able to enhance this transcriptional induction, which is likely to contribute to maintain the caspase cascade activation at a low level.

Immortal Stat3 ${ }^{C / C}$ MEFs display STAT3-dependent aerobic glycolysis. We recently showed that constitutively active STAT3 promotes aerobic glycolysis in both primary Stat3 ${ }^{C / C}$ MEFs and in STAT3-dependent human tumor cell lines via the transcriptional upregulation of $H$ iff- $1 \alpha .{ }^{14}$ To assess glucose metabolism in the immortalized cell lines, a number of glycolysis parameters were evaluated. Similar to the primary cells, immortal Stat3 ${ }^{C / C}$ MEFs displayed increased levels of Hif- $1 \alpha$ and of several of its target genes known to be involved in glycolysis such as pyruvate dehydrogenase kinase-1, lactate dehydrogenase-a and phospho-fructokinase-1 (Figure 4a). Accordingly, immortal Stat3 ${ }^{\mathrm{C} / \mathrm{C}}$ MEFs produced higher lactate levels, thus confirming enhanced aerobic glycolysis (Figure 4b). Both lactate production and the Hif- $1 \alpha$ level were dependent on STAT3 activity, as they were normalized by STAT3 silencing. (Figures 4c and d).

p53 levels or activity are not altered in the Stat $3^{\mathrm{c} / \mathrm{C}}$ MEFs. STAT3 is known to inhibit the expression of p53, which in turn directly regulates glycolysis and mitochondrial 
a

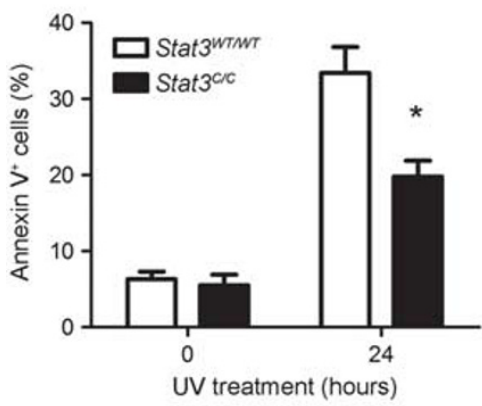

UV
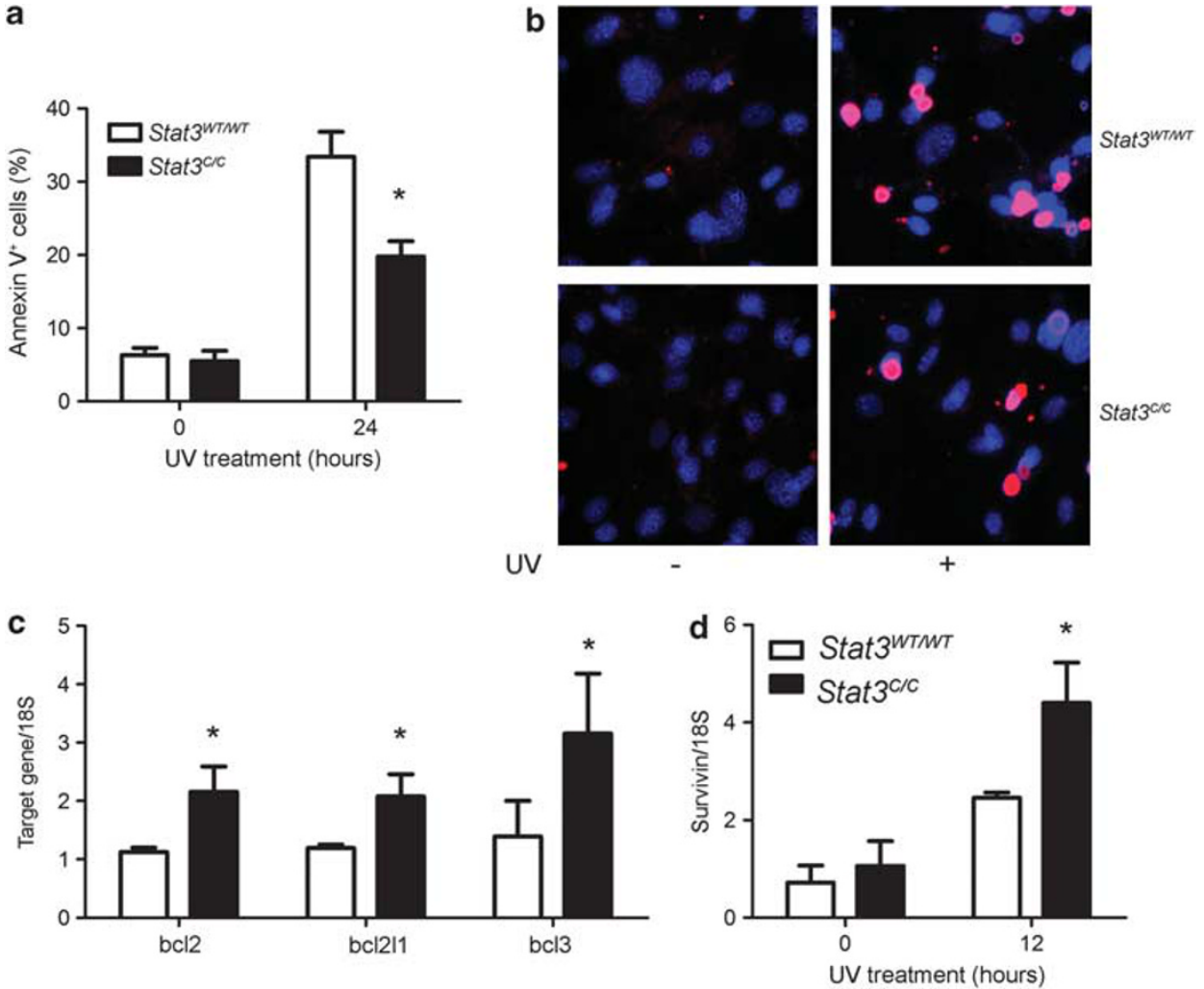

Figure 3 Immortal Stat $3^{C / C}$ MEFs are protected from apoptotic stimuli. (a) Stat3 ${ }^{C / C}$ (black bars) or ${ }^{W T W T}$ (white bars) MEFs were treated with UV-B $\left(10 \mathrm{~J} / \mathrm{m}^{2}\right.$ ) and stained with Annexin $V$ after $24 \mathrm{~h}$, followed by quantification of positive cells by flow cytometry. $N=3$. ${ }^{*} P \leq 0.05$. (b) Same as in a, followed by staining for active CASPASE-3 (red). Nuclei were stained in blue by Hoescht. (c and $\mathbf{d}$ ) The indicated mRNAs were measured by Taqman RT-PCR in Stat3 ${ }^{\text {C/C }}$ (black bars) or ${ }^{\text {WTWT }}$ (white bars) MEFs. N=4. ${ }^{*} P \leq 0.05$. (d) Cells as in a were UV-irradiated followed by RNA extraction and RT-PCR analysis after $12 \mathrm{~h}$

a

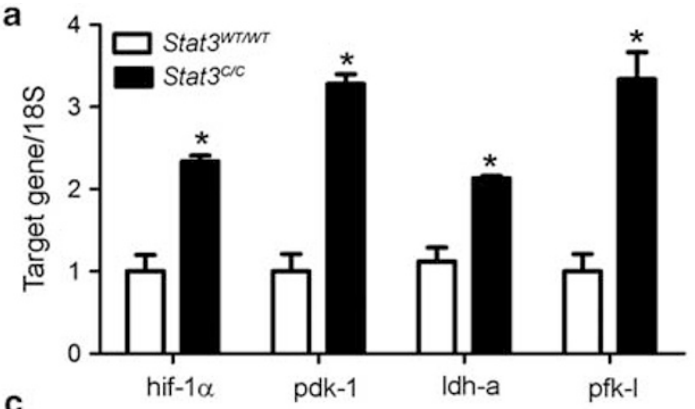

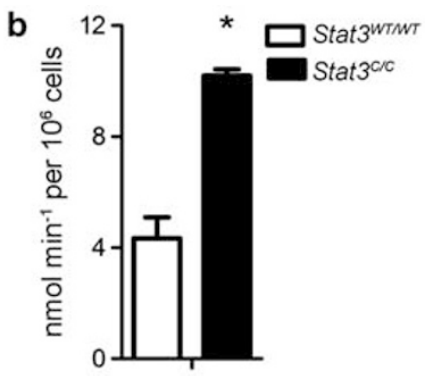
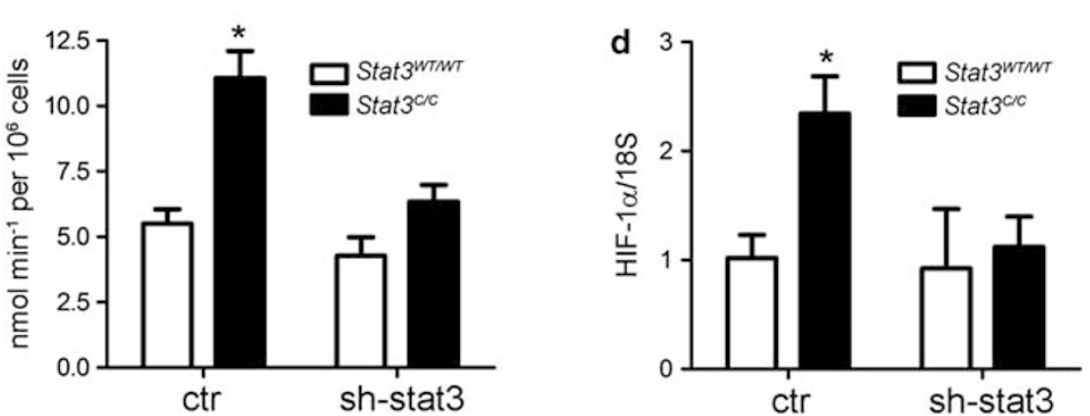

Figure 4 Aerobic glycolysis in Stat3 ${ }^{C / C}$ MEFs. Stat3 ${ }^{C / C}$ (black bars) or ${ }^{\text {WTWT }}$ (white bars) MEFs were assessed for mRNA levels of the indicated genes by Taqman RTPCR (a and $\mathbf{d}$ ) or for their accumulation of lactate in the medium $12 \mathrm{~h}$ after seeding (b and $\mathbf{c})$. Lactate production $(\mathbf{c})$ and Hif- $1 \alpha$ levels (d) were also measured upon Stat3 silencing (sh-stat3). $N=6 .{ }^{*} P \leq 0.01$ 
respiration via some of its target genes such as Sco2 (synthesis of cytochrome $c$ oxidase 2) and Tigar (Tp53-induced glycolysis and apoptosis regulator). ${ }^{27,28}$ Thus, its downregulation may well mediate both resistance to apoptosis and enhanced glycolysis. However, p53 protein levels were not significantly different between Stat ${ }^{W T / W T}$ and Stat $3^{C / C}$ cells (Figure 5a). Accordingly, the mRNA levels of p53 target genes such as p21, Sco2 and Tigar were similar in cells of the two genotypes (Figure $5 \mathrm{~b}$ ). Thus, altered p53 activity cannot apparently explain either the glycolytic phenotype or the protection from apoptosis of the Stat $3^{C / C}$ cells, as we additionally observed normal induction of p21, Sco2 and Tigar expression upon UV treatment (Figure 5c).

Hif-1 $\alpha$ silencing impairs aerobic glycolysis but only partially reverts the transformed phenotype of immortalized Stat ${ }^{C / C}$ MEFs. Hif- $1 \alpha$ is often upregulated in cancer, particularly in late-stage tumors, where it helps protecting cells from hypoxia by activating glycolysis and stimulating angiogenesis. Hif- $1 \alpha$ silencing downregulated aerobic glycolysis in primary Stat $3^{\mathrm{C} / \mathrm{C}} \mathrm{MEFs}$, relieving their glucose dependence. Interfering with Hif- $1 \alpha$ expression dramatically reduced the transcription of glycolysis-related genes and the production of lactate, confirming that indeed STAT3-dependent aerobic glycolysis is mediated by Hif- $1 \alpha$ also in the immortal Stat3 ${ }^{C / C}$ MEFs (Figures $6 a$ and b). In contrast to what observed in the primary cells, however, $\mathrm{Hif}-1 \alpha$ silencing also restored mitochondrial activity, as measured by mitochondrial- $\mathrm{Ca}^{2+}$ uptake, to levels similar to those of the wild-type cells (Figure 6c). Additionally, silencing of Hif- $1 \alpha$ reduced the proliferation rates of immortal Stat $3^{C / C}$ cells (data not shown), and only partially reversed the transformation phenotype. Thus, the ability of immortal
Hif- $1 \alpha$-silenced Stat3 ${ }^{C / C}$ MEFs to form foci and soft agar colonies, as well as their in vivo growth rates, were significantly reduced but not abolished (Figures $6 \mathrm{~d}$ and e). These data suggest that the oncogenic properties of a constitutively active STAT3 partly rely on Hif- $1 \alpha$ upregulation and the consequent metabolic switch towards aerobic glycolysis.

\section{Discussion}

Constitutive, oncogenic STAT3 activity is believed to stem from the aberrant activation of many oncogenic pathways, which ultimately induce its tyrosine phosphorylation. ${ }^{1}$ The prooncogenic role of STAT3 was shown for the first time by the finding that overexpression of the constitutively active mutant form, STAT3C, can transform cultured cells. ${ }^{7,8}$ Further, in vivo, overexpression of STAT3C was shown to enhance malignant progression of skin tumors ${ }^{29}$ and trigger the onset of lung adenocarcinomas. ${ }^{30}$ However, STAT3 activation in tumors most often does not entail increased protein levels, in keeping with the idea that oncogenic STAT3 activation is the leading mechanism ${ }^{31}$ and suggesting that overexpression is probably not the best experimental system to assess the mechanisms of STAT3-mediated tumorigenesis.

We recently showed that expression of STAT3C at physiological levels can cooperate with the Neu oncogene to drive invasive breast cancer, ${ }^{13}$ and that STAT3C knock-in MEFs display features of transformed cells such as increased proliferation, resistance to apoptosis and senescence, and aerobic glycolysis. ${ }^{14}$ Here, we show for the first time that preexisting physiological levels of constitutively active STAT3 are sufficient to transform primary cells to full malignancy following immortalization in a 3T3 protocol, suggesting that low and chronic activity of STAT3 may suffice as a first hit in
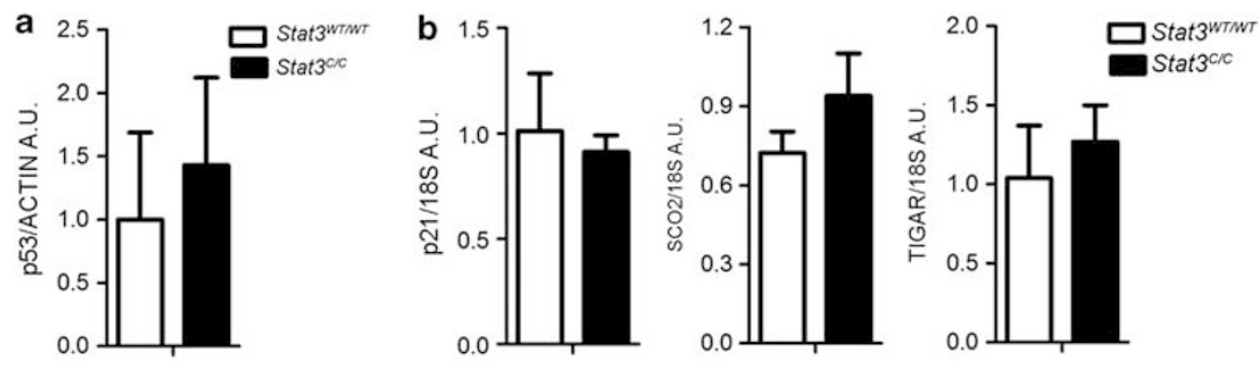

C
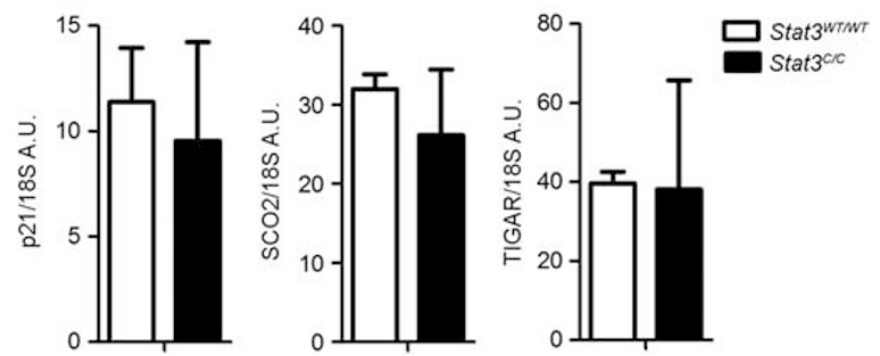

Figure 5 Similar p53 levels and activity in Stat3 ${ }^{C / C}$ or Stat3 ${ }^{W T W T}$ MEFs. Whole protein or RNA extracts were prepared from cells of the indicated genotypes either untreated (a and $\mathbf{b}$ ) or subjected to UV irradiation (c) and analyzed as follows. (a) p53 levels were measured by western blot; ACTIN was used as an internal control. (b) p21, Sco2 and Tigar mRNAs were measured by Taqman RT-PCR in untreated cells, or in UV-irradiated cells (c), where values represent fold induction with respect to untreated cells. The data are mean \pm S.E.M. of at least two independent experiments on each of the cell lines (C1 and C2) 
a

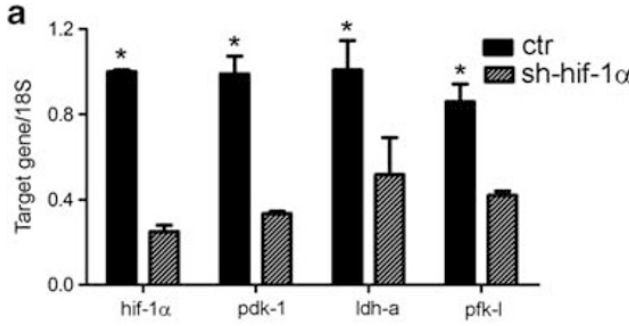

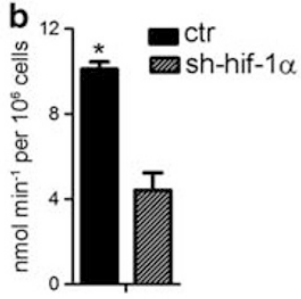

C

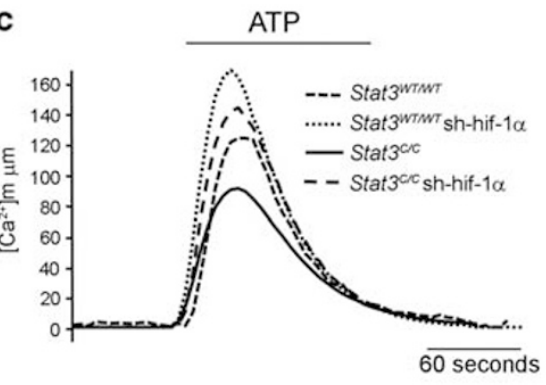

d

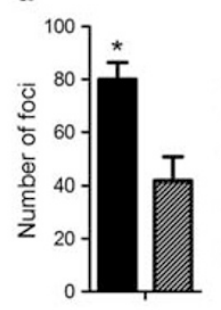

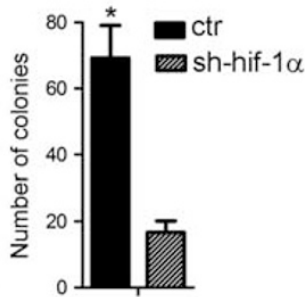

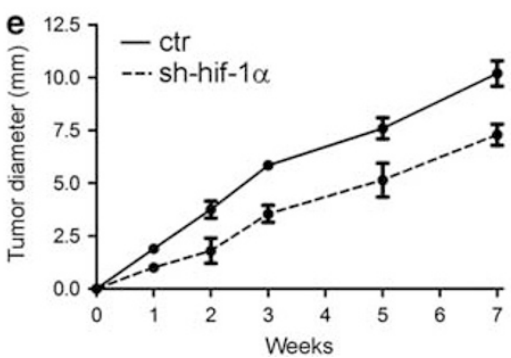

Figure 6 Hif- $1 \alpha$ silencing blunts the oncogenic properties of Stat3 ${ }^{C / C}$ MEFs. Stat3 ${ }^{C / C}$ MEFs either silenced for Hif- $1 \alpha$ (striped bars) or infected with a control virus (black bars) were assessed for mRNA levels of the indicated genes by Tagman RT-PCR (a) or for their accumulation of lactate in the medium $12 \mathrm{~h}$ after seeding (b). (c) MEFs of the indicated genotypes were transduced with a mitochondria-targeted aequorin (AEQ), and aequorin activity was measured upon challenging with $100 \mu \mathrm{M}$ ATP. (d and e) Stat3 ${ }^{\mathrm{C} / \mathrm{C}}$ MEFs either silenced or not for Hif-1 $\alpha$ were tested for their ability to form foci and colonies in soft agar (d), or tested for in vivo growth (e) as described in the legend to Figure 1. $N=4 .{ }^{*} P \leq 0.01$

multistep carcinogenesis. This finding is of particular relevance for the emerging key role of STAT3 in inflammationdriven cancer. It has been shown that chronic inflammation, entailing a positive loop between the transcription factor $\mathrm{NF}-\kappa \mathrm{B}$, the pro-inflammatory cytokine IL-6 and the IL-6activated STAT3, is a highly predisposing condition for cancer, particularly in the colon, liver and breast. ${ }^{32}$ In addition, to generate a favorable niche for the growth of tumor cells via the production of pro-oncogenic signals, our data show now that cells exposed to chronic IL-6 signaling, displaying continuous STAT3 activation, can behave like cells that have undergone a first oncogenic mutation. These cells will therefore be exquisitely sensitive to further mutagenic events, which are favored by the inflammatory microenvironment that contains cytokines, growth factors and reactive oxygen species.

The mechanism through which constitutively active STAT3 sensitizes cells to tumorigenic transformation is probably many-fold and observed in either our Stat $3^{C / C}$ primary ${ }^{14}$ or immortalized MEFs: first, protection from apoptosis; second, delayed senescence; third, increased proliferation and fourth, increased glycolytic metabolism. Protection from apoptosis, induced by different stress stimuli, correlates with the increased expression of anti-apoptotic genes. Delayed senescence and increased proliferation likely correlate with a metabolic switch towards aerobic glycolysis. Aerobic glycolysis was recently proposed to give an advantage to rapidly proliferating cells, allowing them to accumulate NADPH and carbon skeletons needed for anabolic reactions as a side-product of glucose consumption and ATP production. ${ }^{24}$ Another important player in oncogenesis is c-myc, which contributes to increased proliferation and aerobic glycolysis, and indeed the levels of c-myc, ${ }^{13}$ a well known STAT3 target, are elevated in the Stat ${ }^{C / C}$ MEFs. ${ }^{33}$

$\mathrm{HIF-1} \alpha$ is often overexpressed in cancer cells, having key roles in critical aspects of cancer biology, including angiogenesis, cell survival, genetic instability, invasion and metastasis, proliferation and metabolism. ${ }^{34}$ Indeed, HIF- $1 \alpha$ is a well known inducer of aerobic glycolysis, and it was not therefore surprising to find that STAT3-induced glycolysis was Hif-1 $\alpha$-dependent. Interestingly, the twofold transcriptional induction of HIF- $1 \alpha$ triggered by constitutively active STAT3 was enough to elevate protein levels without inducing protein stabilization, thought to be essential for enhanced HIF-1 $\alpha$ activity. ${ }^{35}$ Similar to their primary counterparts, immortal Stat $3^{\mathrm{C} / \mathrm{C}}$ cells show HIF-1 $\alpha$-dependent aerobic glycolysis. Interestingly, however, HIF-1 $\alpha$ silencing reduced, but did not abolish, several transformed phenotypes. Thus, 
STAT3-induced HIF-1 $\alpha$ overexpression and aerobic glycolysis are important contributors to STAT3-mediated tumorigenesis.

MEF cells immortalization usually entails loss of function of either the p53 or the ARF oncosuppressors. ${ }^{36}$ In particular p53, known to be repressed by STAT3 and to mediate both c-myc and HIF-1 $\alpha$ downregulation while regulating mitochondrial respiration, ${ }^{28}$ was a good candidate to mediate at least part of the transformed phenotype of Stat $3^{C / C}$ cells. However, our results suggest that p53 levels and activity are similar in cells of the two genotypes, thus ruling out this hypothesis.

Interestingly, RAS overexpression induced senescence in Stat $3^{C / C}$ primary MEFs, similar to its effect in wild-type cells ${ }^{37}$ (data not shown), possibly because RAS and STAT3 act in the same signaling cascade. Indeed, STAT3 is phosphorylated on Serine 727 following RAS signaling, and it was shown to support RAS-mediated transformation via mitochondrial localization and function. ${ }^{15}$ By induced Serine phosphorylation, RAS might therefore increase STAT3C localization to mitochondria, thus reducing its nuclear concentration and activity. We have previously shown that nuclear constitutively active STAT3 restrained mitochondrial metabolism in both primary MEFs and STAT3-dependent tumor cells by a HIF-1 $\alpha$-independent mechanism. ${ }^{14}$ Also 3T3 Stat3 ${ }^{C / C}$ cells showed decreased mitochondrial activity, which could be rescued by $\mathrm{HIF}-1 \alpha$ silencing. This may reflect the intrinsic heterogeneity in the transformation process, where tumor cells may come to rely more heavily on different pathways upon transformation.

In conclusion, our data suggest that aberrantly constitutively active STAT3 can act as a first hit during tumorigenic transformation supporting tumor survival, growth and metabolism, strengthening its definition as an oncogene. Thus, drugs inhibiting STAT3 activity might be beneficial not only in the therapy of cancer but also in prophylactic treatments aimed at preventing the onset of inflammationdriven tumorigenesis.

\section{Materials and Methods \\ Mice, MEFs preparation, cell lines derivation and culture. Stat3C/C mice ${ }^{13}$ were maintained in the transgenic unit of the Molecular Biotechnology Center (University of Turin). Procedures were conducted in conformity with national and international laws and policies as approved by the Faculty Ethical Committee. Embryos were dissected 13.5 days post coitum for MEF derivation. 3 T 3 cell lines were prepared as previously described. ${ }^{25}$ 3T3 MEFs were grown in DMEM with GLUTAMAX (Dulbecco's modified Eagle medium; Gibco-BRL, Carlsbad, CA, USA), supplemented with $10 \%$ (V/v) heat-inactivated fetal calf serum (Gibco-BRL), $100 \mathrm{U} / \mathrm{ml}$ penicillin, $100 \mu \mathrm{g} / \mathrm{ml}$ streptomycin.}

Cell proliferation, FFA and soft agar. For cell proliferation rate, $1.5 \times 10^{5}$ cells were seeded in six-well plates and counted at the indicated times using the Countess Automated Cell Culture (Invitrogen, Carlsbad, CA, USA). Population doublings were calculated using the formula $P D=t \log 2 / \log N t-\log$ No.

For the FFA, $30 \times 10^{4}$ cells were seeded in a $10 \mathrm{~cm}$ plate and the media refreshed every 2-3 days. After 15 days, cells were fixed in cold methanol and stained with methyl violet (Sigma-Aldrich, St. Louis, MO, USA). Plates were scanned and the number of colonies was calculated using the software ImageJ (http://rsbweb.nih.gov/ij/).

For soft agar assay, $2 \times 10^{4}$ cells were plated in $0.35 \%$ agar in complete growth medium in $3.5 \mathrm{~cm}$ wells. Colonies were counted 30 days after seeding.

Lentiviral infection. pLKO vectors carrying either scrambled or shRNA-HIF$1 \alpha$ sequences (Open Biosystems, Huntsville, AL, USA) and pLVTH-GFP carrying either scrambled or shRNA-STAT3 sequences ${ }^{38}$ were packaged by transfecting $293 \mathrm{~T}$ cells and used to infect cells for $24 \mathrm{~h}$, followed by puromycin selection for $48 \mathrm{~h}$.

Subcutaneous tumors. Female CD-1 mice 5-7 weeks of age (Charles River Laboratories, Inc., Wilmington, MA, USA) were s.c. implanted with 3 T3 cells. Cells were harvested using trypsin and washed with PBS. In all, 106 cells were resuspended in $200 \mu \mathrm{l}$ PBS and injected s.c. into the left flank of mice. All mice were monitored two times weekly for body weight. s.c. tumors were measured with a caliper weekly, and mice were killed whenever the tumor exceeded $10 \mathrm{~mm}$ of diameter.

Real time-PCR. Total RNA was prepared with the PureLink Micro-to-Midi total RNA Purification System (Invitrogen). qRT-PCR reactions were performed using the Universal Probe Library system (Roche Italia, Monza, Italy). The 18s rRNA pre-developed TaqMan assay (Applied Biosystems, Carlsbad, CA, USA) was used as an internal control. For primers and probes see Supplementary Information.

Immunofluorescence. Cells plated on glass coverslips were washed in PBS, fixed in $4 \%$ paraformaldehyde, quenched with $50 \mathrm{mmol} / \mathrm{l}$ ammonium chloride, permeabilized with $0.3 \%$. Triton X-100 in PBS, saturated with $3 \%$ bovine serum albumin, and incubated with primary antibodies at room temperature for $1 \mathrm{~h}$, followed by rhodamine-labeled secondary antibodies (Sigma-Aldrich Italia, Milano, Italy) and then by Hoechst-dye. An Axiovert 200M Zeiss microscope (Carl Zeiss, Milan, Italy) for optical sectioning was used.

Western blots. Total and nuclear protein extracts were obtained as previously described. ${ }^{14}$ Samples were fractionated on SDS-PAGE and transferred to a polyvinylidene difluoride membrane (Millipore, Billerica, MA, USA).

Lactate measurements. In all, $10^{5}$ cells were seeded and the media were refreshed $12 \mathrm{~h}$ later. Lactate was measured in cell supernatants $3 \mathrm{~h}$ after changing medium using a Lactate Colorimetric Assay Kit (Abcam, Cambridge, MA, USA). Data were normalized to final cell counts.

Mitochondrial calcium measurements. Cells were grown on glass coverslips at $50 \%$ confluence and infected with the adenovirus expressing the appropriate aequorin chimera as previously described. ${ }^{14}$ Measurements were carried out in KRB $\left(125 \mathrm{mM} \mathrm{NaCl}, 5 \mathrm{mM} \mathrm{KCl}, 1 \mathrm{mM} \mathrm{MgSO}_{4}, 1 \mathrm{mM} \mathrm{Na}_{2} \mathrm{HPO}_{4}\right.$, $5.5 \mathrm{mM}$ glucose, $20 \mathrm{mM} \mathrm{NaHCO}_{3}, 2 \mathrm{mM} \mathrm{I-glutamine}$ and $20 \mathrm{mM}$ HEPES, pH 7.4, supplemented with $1 \mathrm{mM} \mathrm{CaCl}_{2}$ ). ATP was added to the same medium. Cells were lysed with $100 \mu \mathrm{M}$ digitonin in a hypotonic $\mathrm{Ca}^{2+}$-rich solution $\left(10 \mathrm{mM} \mathrm{CaCl}_{2}\right.$ in $\mathrm{H}_{2} \mathrm{O}$ ), thus discharging the remaining aequorin pool. The light signal was collected and calibrated into $\left[\mathrm{Ca}^{2+}\right]$ values.

In vitro cell death. Cells were irradiated with $10 \mathrm{~J} / \mathrm{m}^{2} \mathrm{UV}$-C or serum-starved for 48-72 $\mathrm{h}$, followed by staining with either Annexin- $\mathrm{V}$ or anti-activated Caspase-3. Annexin-V emission were detected in the green channel $(525 \mathrm{~nm})$ and propidium iodide in the red channel $(575 \mathrm{~nm})$ following excitation by a $488 \mathrm{~nm}$ laser on a FACS Calibur cytometer (Beckton, Dickinson and Company, Franklin Lakes, NJ, USA).

Statistical analysis. An unpaired $t$-test was used to calculate the $P$-value for two groups, while the $P$-value on a response affected by two factors was calculated with a two-way ANOVA.

\section{Conflict of Interest}

The authors declare no conflict of interest.

Acknowledgements. We thank M Wieckowski for helpful discussion and for critically reading the manuscript. This work was supported by the Italian Cancer Research Association (AIRC), the Italian Ministry for University and Research (FIRB and MIUR), the Association for International Cancer Research (AICR), Telethon, the Italian Multiple Sclerosis Foundation and the Italian Ministry of Health.

1. Turkson J, Jove R. STAT proteins: novel molecular targets for cancer drug discovery. Oncogene 2000; 19: 6613-6626.

2. Siddiquee K, Zhang S, Guida WC, Blaskovich MA, Greedy B, Lawrence HR et al. Selective chemical probe inhibitor of Stat3, identified through structure-based virtual screening, induces antitumor activity. Proc Natl Acad Sci USA 2007; 104: 7391-7396. 
3. Schindler C, Levy DE, Decker T. JAK-STAT signaling: from interferons to cytokines J Biol Chem 2007; 282: 20059-20063.

4. Yu CL, Meyer DJ, Campbell GS, Larner AC, Carter-Su C, Schwartz J et al. Enhanced DNA-binding activity of a Stat3-related protein in cells transformed by the Src oncoprotein. Science 1995; 269: 81-83.

5. Bromberg JF, Horvath CM, Besser D, Lathem WW, Darnell Jr JE. Stat3 activation is required for cellular transformation by v-src. Mol Cell Biol 1998; 18: 2553-2558.

6. Silva CM. Role of STATs as downstream signal transducers in Src family kinase-mediated tumorigenesis. Oncogene 2004; 23: 8017-8023.

7. Bromberg JF, Wrzeszczynska MH, Devgan G, Zhao Y, Pestell RG, Albanese $\mathrm{C}$ et al. Stat3 as an oncogene. Cell 1999; 98: 295-303.

8. Dechow TN, Pedranzini L, Leitch A, Leslie K, Gerald WL, Linkov I et al. Requirement of matrix metalloproteinase- 9 for the transformation of human mammary epithelial cells by Stat3-C. Proc Natl Acad Sci USA 2004; 101: 10602-10607.

9. Pilati C, Amessou M, Bihl MP, Balabaud C, Nhieu JT, Paradis V et al. Somatic mutations activating STAT3 in human inflammatory hepatocellular adenomas. J Exp Med 2011; 208 $1359-1366$

10. Bromberg J, Wang TC. Inflammation and cancer: IL-6 and STAT3 complete the link. Cancer Cell 2009; 15: 79-80.

11. Yu H, Pardoll DM, Jove R. STATs in cancer inflammation and immunity: a leading role for STAT3. Nat Rev Cancer 2009; 9: 798-809.

12. Pensa S, Regis G, Boselli D, Novelli F, Poli V. STAT1 and STAT3 in tumorigenesis: two sides of the same coin? In: Stephanou A (eds). JAK-STAT Pathway in Disease. Landes Bioscience: Austin, 2009, pp 100-121.

13. Barbieri I, Pensa S, Pannellini T, Quaglino E, Maritano D, Demaria M et al. Constitutively active Stat3 enhances neu-mediated migration and metastasis in mammary tumors via upregulation of Cten. Cancer Res 2010; 70: 2558-2567.

14. Demaria M, Giorgi C, Lebiedzinska M, Esposito G, D'Angeli L, Bartoli A et al. A STAT3 mediated metabolic switch is involved in tumour transformation and STAT3 addiction. Aging (Albany NY) 2010; 2: 823-842.

15. Gough DJ, Corlett A, Schlessinger K, Wegrzyn J, Larner AC, Levy DE. Mitochondrial STAT3 supports Ras-dependent oncogenic transformation. Science 2009; 324 1713-1716.

16. Wegrzyn J, Potla R, Chwae YJ, Sepuri NB, Zhang Q, Koeck T et al. Function of mitochondrial Stat3 in cellular respiration. Science 2009; 323: 793-797.

17. Demaria M, Poli V. From the nucleus to the mitochondria and back: the odyssey of a multitask STAT3. Cell Cycle 2011; 10: 3221-3222.

18. Semenza GL. Defining the role of hypoxia-inducible factor 1 in cancer biology and therapeutics. Oncogene 2010; 29: 625-634.

19. Kaelin Jr WG, Ratcliffe PJ. Oxygen sensing by metazoans: the central role of the HIF hydroxylase pathway. Mol Cell 2008; 30: 393-402.

20. Kronblad A, Jirstrom K, Ryden L, Nordenskjold B, Landberg G. Hypoxia inducible factor1alpha is a prognostic marker in premenopausal patients with intermediate to highly differentiated breast cancer but not a predictive marker for tamoxifen response. Int J Cancer 2006; 118: 2609-2616.
21. Giatromanolaki A, Sivridis E, Kouskoukis C, Gatter KC, Harris AL, Koukourakis Ml. Hypoxia-inducible factors 1alpha and 2alpha are related to vascular endothelial growth factor expression and a poorer prognosis in nodular malignant melanomas of the skin. Melanoma Res 2003; 13: 493-501.

22. Nanni S, Benvenuti V, Grasselli A, Priolo C, Aiello A, Mattiussi S et al. Endothelial NOS, estrogen receptor beta, and HIFs cooperate in the activation of a prognostic transcriptional pattern in aggressive human prostate cancer. J Clin Invest 2009; 119: 1093-1108.

23. Warburg 0 . On respiratory impairment in cancer cells. Science 1956; 124: 269-270.

24. Vander Heiden MG, Cantley LC, Thompson CB. Understanding the Warburg effect: the metabolic requirements of cell proliferation. Science 2009; 324: 1029-1033.

25. Todaro GJ, Green H. Quantitative studies of the growth of mouse embryo cells in culture and their development into established lines. J Cell Biol 1963; 17: 299-313.

26. Krontiris TG, Cooper GM. Transforming activity of human tumor DNAs. Proc Natl Acad Sci USA 1981; 78: 1181-1184.

27. Bensaad K, Tsuruta A, Selak MA, Vidal MN, Nakano K, Bartrons R et al. TIGAR, a p53-inducible regulator of glycolysis and apoptosis. Cell 2006; 126: 107-120.

28. Matoba S, Kang JG, Patino WD, Wragg A, Boehm M, Gavrilova $O$ et al. p53 regulates mitochondrial respiration. Science 2006; 312: 1650-1653.

29. Chan KS, Sano S, Kataoka K, Abel E, Carbajal S, Beltran L et al. Forced expression of a constitutively active form of Stat3 in mouse epidermis enhances malignant progression of skin tumors induced by two-stage carcinogenesis. Oncogene 2008; 27: 1087-1094.

30. Li Y, Du H, Qin Y, Roberts J, Cummings OW, Yan C. Activation of the signal transducers and activators of the transcription 3 pathway in alveolar epithelial cells induces inflammation and adenocarcinomas in mouse lung. Cancer Res 2007; 67: 8494-8503.

31. Okamoto W, Okamoto I, Arao T, Yanagihara K, Nishio K, Nakagawa K. Differential roles of STAT3 depending on the mechanism of STAT3 activation in gastric cancer cells. Br J Cancer 2011; 105: 407-412.

32. Li N, Grivennikov SI, Karin M. The unholy trinity: inflammation, cytokines, and STAT3 shape the cancer microenvironment. Cancer Cell 2011; 19: 429-431.

33. Kiuchi N, Nakajima K, Ichiba M, Fukada T, Narimatsu M, Mizuno K et al. STAT3 is required for the gp130-mediated full activation of the c-myc gene. J Exp Med 1999; 189: 63-73.

34. Semenza GL. Targeting HIF-1 for cancer therapy. Nat Rev Cancer 2003; 3: 721-732.

35. Maxwell PH, Ratcliffe PJ. Oxygen sensors and angiogenesis. Semin Cell Dev Biol 2002; 13: 29-37.

36. Zindy F, Eischen CM, Randle DH, Kamijo T, Cleveland JL, Sherr CJ et al. Myc signaling via the ARF tumor suppressor regulates p53-dependent apoptosis and immortalization. Genes Dev 1998; 12: 2424-2433.

37. Serrano M, Lin AW, McCurrach ME, Beach D, Lowe SW. Oncogenic ras provokes premature cell senescence associated with accumulation of p53 and p16INK4a. Cell 1997; 88: $593-602$.

38. Piva R, Pellegrino E, Mattioli M, Agnelli L, Lombardi L, Boccalatte $F$ et al. Functional validation of the anaplastic lymphoma kinase signature identifies CEBPB and BCL2A1 as critical target genes. J Clin Invest 2006; 116: 3171-3182.

Supplementary Information accompanies the paper on Cell Death and Differentiation website (http://www.nature.com/cdd) 